University of Nebraska - Lincoln

DigitalCommons@University of Nebraska - Lincoln

May 2005

\title{
Relaxation in magnetic nanostructures
}

\author{
Ralph Skomski \\ University of Nebraska-Lincoln, rskomski2@unl.edu \\ J. Zhou \\ University of Nebraska - Lincoln \\ David J. Sellmyer \\ University of Nebraska-Lincoln, dsellmyer@unl.edu
}

Follow this and additional works at: https://digitalcommons.unl.edu/physicssellmyer

Part of the Physics Commons

Skomski, Ralph; Zhou, J.; and Sellmyer, David J., "Relaxation in magnetic nanostructures" (2005). David Sellmyer Publications. 14.

https://digitalcommons.unl.edu/physicssellmyer/14

This Article is brought to you for free and open access by the Research Papers in Physics and Astronomy at DigitalCommons@University of Nebraska - Lincoln. It has been accepted for inclusion in David Sellmyer Publications by an authorized administrator of DigitalCommons@University of Nebraska - Lincoln. 


\title{
Relaxation in magnetic nanostructures
}

\author{
R. Skomski, ${ }^{\text {a) }}$ J. Zhou, and D. J. Sellmyer \\ Department of Physics and Astronomy and Center for Materials Research and Analysis, University \\ of Nebraska, Lincoln, Nebraska 68588
}

(Presented on 9 November 2004; published online 28 April 2005)

\begin{abstract}
The damping of magnetization processes in nanostructures is investigated by Fokker-Planck-type model calculations and quantum-mechanical considerations based on Fermi's golden rule. In the absence of energy barriers, the problem reduces to a magnetic diffusion equation with a particle-size-dependent diffusivity which is indirectly proportional to the relaxation-time parameter $\tau_{\mathrm{o}}$. For small particles, the relaxation time is proportional to the particle volume, but when the particle size reaches a few nanometers, it approaches a constant bulk value. (C) 2005 American Institute of Physics. [DOI: 10.1063/1.1847854]
\end{abstract}

\section{INTRODUCTION}

The relaxation behavior of magnetic nanostructures is of interest in many areas of advanced technology, including magnetic recoding and sensor applications, and a key input in numerical simulations. An important and largely unsolved issue is the origin of damping-parameter $\lambda$, which determines the relaxation time of the spin precession. ${ }^{1,2}$

An exact way of treating relaxation starts from the Liouville-von Neumann equation and includes both relevant magnetic and irrelevant heat-bath degrees of freedom. ${ }^{3-5}$ For sufficiently slow magnetization processes, there is a separation of macroscopic and microscopic time scales, and the relaxation time $\tau$ and related parameters, such as $\lambda$, have a well-defined meaning. In addition, the separation of time scales yields a Landau-Lifshitz-type precession term and a Langevin force responsible for the thermal activation over energy barriers, which are observed as magnetic viscosity and as the sweep-rate dependence of the coercivity. ${ }^{6}$

The magnetization precession, the damping, and the thermal forces depend on the magnetic system in consideration. The strength of the thermal forces is given by the temperature of the heat bath, ${ }^{7}$ whereas the precession is, essentially, a deterministic zero-temperature property. The damping reflects the interaction between magnetic and heatbath degrees of freedom, as described by Fermi's golden rule

$$
W_{i j}=\frac{2 \pi}{\hbar}\left|\left\langle\Psi_{i}|V| \Psi_{j}\right\rangle\right|^{2} \delta\left(E_{i}-E_{j}\right) .
$$

Here $W_{i j}$ is the transition rate between two quantum states $i$ and $j$, and $\left\langle\Psi_{i}|V| \Psi_{j}\right\rangle$ is the matrix element between the two states. These matrix elements lead to the time-dependent decay of the original modes. It conserves the total energy of the system but includes energy redistributions between different subsystems. Fermi's golden rule applies to a large variety of physical transitions and includes, for example, transitions between magnetic and phononic degrees of freedom. ${ }^{8-10}$

The transition rates $W_{i j}$ determine the dynamics of the system. Simplifying somewhat, there are descriptions based on three different types of equations: (i) master or rate equa-

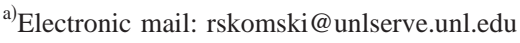

tions, (ii) Fokker-Planck or generalized diffusion equations, and (iii) Langevin or random-force equations. These equations are physically largely equivalent, ${ }^{7,11}$ and their application to magnetic systems is well established. ${ }^{12-14}$ For example, the Arrhenius or Néel-Brown law

$$
\tau=\tau_{0} \exp \left(E_{a} / k_{B} T\right),
$$

is a low-temperature solution of the Fokker-Planck equation. ${ }^{5,15,16}$ Here $\tau$ is the relaxation time for a thermally activated process over an energy barrier $E_{a}$ and $\tau_{\mathrm{o}} \sim 10^{-9} \mathrm{~s}$ is the relaxation time in the absence of energy barriers. As the respective Landau-Lifshitz and Gilbert damping parameters $\lambda$ and $\eta,{ }^{17} 1 / \tau_{\mathrm{o}}$ is essentially proportional to $W_{i j}$, but the quantification of relaxation parameters has remained a complicated issue, and $\tau_{\mathrm{o}}$ is usually considered as a phenomenological parameter. Here we use the magnetic Fokker-Planck equation to explain $\tau_{\mathrm{o}}$ in terms of spin diffusion.

\section{CALCULATION AND RESULTS}

\section{A. Damped precession and diffusion in spin space}

Consider a magnetic nanoparticle containing $N$ atoms. When the particles are sufficiently small, the spins are strongly exchange coupled, and the particle's magnetization has the character of a macrospin of magnitude $S N$. The magnetization reversal can then be rationalized in terms of magnetization angles $\phi$ and $\theta$. The magnetization dynamics has two aspects: a damped precession towards the local effective field and a random thermal motion of the magnetization vector. Figure 1 illustrates the two limits. The damped precession is, essentially, a zero-temperature phenomenon, whereas the randomness of (b) reflects the interaction with the heat bath. Note the opposite direction of the motions: the relaxation moves towards a local energy minimum, whereas the random motion is diffusive and generally points away from the starting point.

Physically, Fig. 1(a) is realized for low temperatures and pronounced effective fields, as encountered in typical resonance experiments. The diffusive regime, Fig. 1(b), is realized in the case of very weak effective fields (spherical particles, no applied field, and zero magnetic anisotropy). Here we are interested in the diffusive regime. Putting $E_{a}=0$ in 

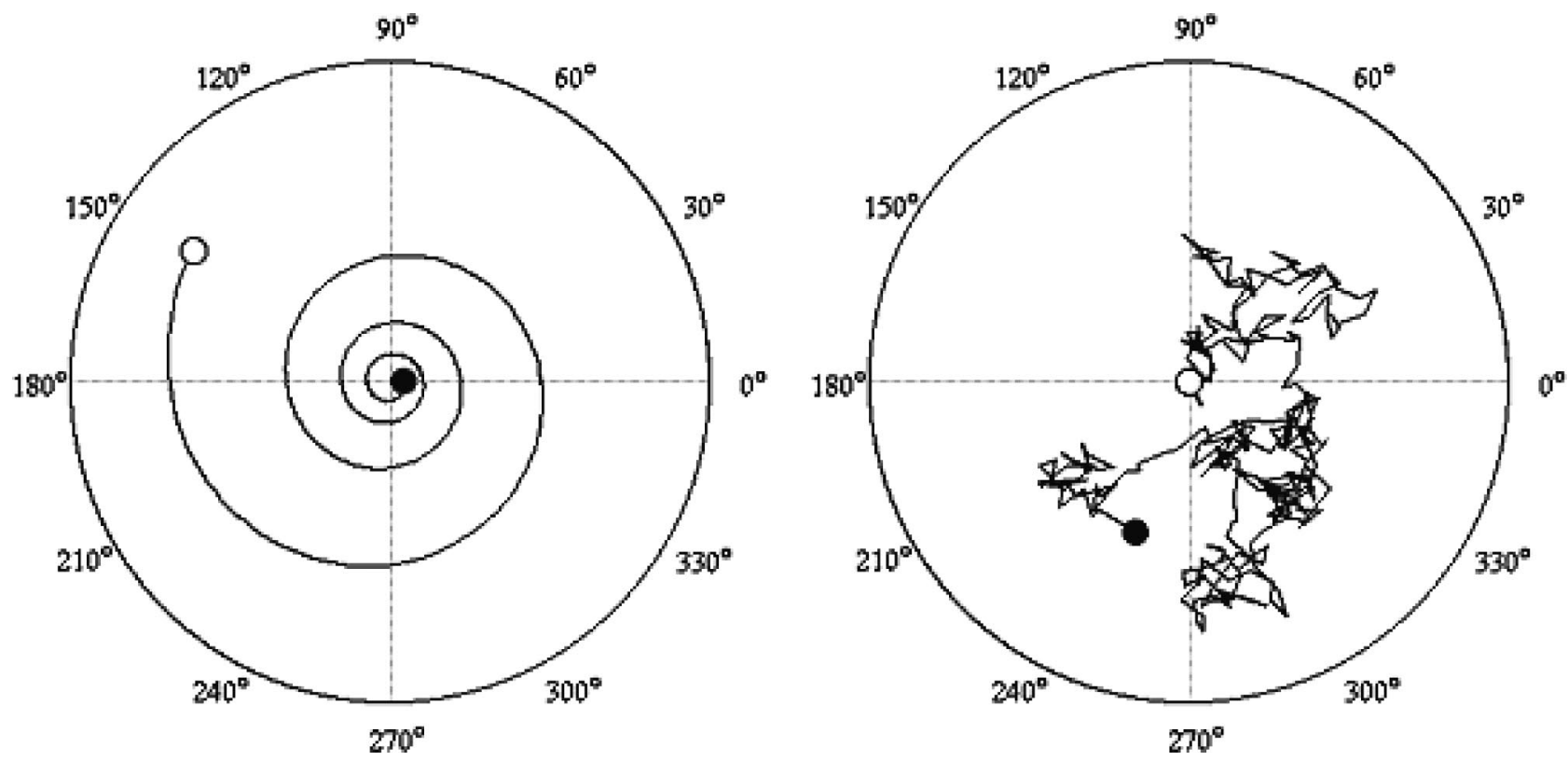

FIG. 1. Magnetization dynamics of a nanoparticle: (a) damped precession and (b) random thermal motion. The curves are simulations for typical but not critical parameters, covering a time of order $0.1 \mathrm{~ns}$. In both polar plots, the direction of the motion is from the white circles to the black circles.

Eq. (2) yields $\tau=\tau_{0}$, so that the solution of the diffusion problem of Fig. 1(b) amounts to the determination of $\tau_{\mathrm{o}}$.

\section{B. Fokker-Planck analysis}

In the absence of an effective field, the Fokker-Planck equation governing the spin diffusion reduces to an ordinary diffusion equation. ${ }^{7,11}$ In the present case, the diffusivity depends on the matrix elements $\left\langle\Psi_{i}|V| \Psi_{i}\right\rangle$ $=\left\langle\psi\left(\phi^{\prime}, \theta^{\prime}\right)|V| \Psi(\phi, \theta)\right\rangle$ between neighboring spin states. Alternatively, the path shown in Fig. 1(b) can be modeled as a random walk where $\theta(t+\delta t)=\theta(t) \pm \varepsilon_{0}$ and $\phi(t+\delta t)$ $=\phi(t) \pm \sin ^{-1}(\theta) \varepsilon_{0}$. In this approach, there are two parameters, $\delta t$ and $\varepsilon_{0}$. The parameter $\varepsilon_{\mathrm{o}}$ is fixed by the requirement that the equilibrium solution of the underlying FokkerPlanck equation reproduces equilibrium statistical mechanics. ${ }^{7}$ Illustratively speaking, the higher the temperature, the larger $\varepsilon_{0}$. The parameter $\delta t \sim W_{i j}^{-1}$ describes the dynamics of the system; it can be interpreted as the time necessary to proceed from $\theta$ and $\phi$ to $\theta^{\prime}$ and $\phi^{\prime}$.

Starting from $\theta=0$ and $\phi=0$, as indicated in Fig. 1(b), we now calculate the magnetization projection $M(t)$ $=M_{\mathrm{o}}\langle\cos [\theta(t)]\rangle$. Since $M(t)$ is independent of $\phi(t)$, we can restrict ourselves to the diffusion of the variable $\theta$. The probability $P(\theta)$ obeys the diffusion equation $\partial P / \partial t$ $=\Gamma_{\text {eff }} \partial^{2} P / \partial \theta^{2}$, where $\Gamma_{\text {eff }}=\varepsilon_{0}^{2} / 2 \delta t$, so that

$$
P(\theta, t)=\frac{1}{\sqrt{4 \pi \Gamma_{\text {eff }} t}} \exp \left(-\frac{\theta^{2}}{4 \Gamma_{\text {eff }} t}\right) .
$$

Evaluating the integral $M(t)=M_{\mathrm{o}} \int P(\theta, t) \cos (\theta) d \theta$, where $\theta$ extends from $-\infty$ to $+\infty$, yields $M(t)=M_{\mathrm{o}} \exp \left(-\Gamma_{\text {eff }} t\right)$. Essentially, this simple result amounts to a functional integration over all possible diffusion paths, Fig. 1(b), and means that the average magnetization projection decays with a relaxation time $\tau_{\mathrm{o}}=1 / \Gamma_{\text {eff }}$.

\section{Relaxation time}

The next step is to relate $\Gamma_{\text {eff }}=1 / \tau_{\mathrm{o}}$ to the properties of the particle. In particular, $\Gamma_{\text {eff }}$ may depend on the number of particles $N$, so that $\Gamma_{\text {eff }}=\Gamma_{\text {eff }}(N)$. For simplicity, we consider $N$ exchange-coupled spins characterized by one rotational degree of freedom $\theta_{i}$ per spin $(i=1, \ldots, N)$. The dynamics of a system described by Eq. (3) is then equivalent to the Langevin equation

$$
\frac{\partial \theta_{i}}{\partial t}=\sqrt{2 \Gamma_{\mathrm{o}}} \xi_{i}(t),
$$

where $\Gamma_{\mathrm{o}}=\Gamma_{\text {eff }}(1)$ and $\xi_{i}(t)$ is a delta-correlated random force obeying $\left\langle\xi_{i}(t)\right\rangle=0$ and $\left\langle\xi_{i}(t) \xi_{j}\left(t^{\prime}\right)\right\rangle=\delta_{i j} \delta\left(t-t^{\prime}\right) .{ }^{7,14}$ Of course, from a quantum-mechanical point of view, Eqs. (3) and (4) are crude approximations for single or few spin systems, ${ }^{18}$ but we are not interested in this limit and assume that $N \gg 1$.

Next, we assume that the $N$ spins are strongly exchange coupled, so that all spins are parallel and therefore $\theta_{i}=\theta$. Adding all contributions $\partial \theta_{i} / \partial t$ in Eq. (4) leads to

$$
N \frac{\partial \theta}{\partial t}=\sqrt{2 \Gamma_{\mathrm{o}} N} \xi_{i}(t) .
$$

In this equation, the square-root dependence of the randomforce term on $N$ originates from $\Sigma_{i} \Sigma_{j}\left\langle\xi_{i}(t) \xi_{j}\left(t^{\prime}\right)\right\rangle=N \delta\left(t-t^{\prime}\right)$. Dividing Eq. (5) by $N$ yields

$$
\frac{\partial \theta}{\partial t}=\sqrt{2 \Gamma_{\mathrm{eff}}} \xi_{i}(t)
$$

where $\Gamma_{\text {eff }}=\Gamma_{\mathrm{o}} / N$. In other words, the square-root dependence on $N$ in the source term of Eq. (5) translates into a $1 / N$ dependence of $\Gamma_{\text {eff }}$.

The same result is obtained by a quantum-mechanical argumentation. Equation (1) involves integrations of the type 


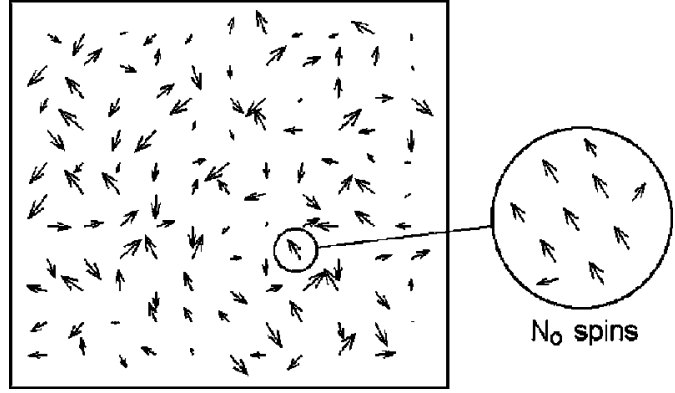

FIG. 2. Cooperative spin blocks. When the size $N$ of the particle becomes too big, then thermal activation leads to the formation of cooperative units of size $N_{\mathrm{o}}$.

$\int \psi^{*}(\mathbf{r}) V(\mathbf{r}) \psi(\mathbf{r}) d V$, and since thermal forces are operative on a local scale, $W_{i j}$ is linear rather than quadratic in the particle size.

The requirement that the Langevin description reproduce equilibrium statistics when applied to an energy landscape implies that $\Gamma_{\text {eff }} \sim T$. Equation (3) shows that $\Gamma_{\text {eff }}$ is, essentially, a diffusion coefficient, so that $\Gamma_{\text {eff }} \sim T$ can be interpreted as a magnetic analogy to the Einstein-Smoluchowski relation $D \sim T$ for the Brownian motion of a particle. As a consequence, we can summarize the above considerations by $\tau_{\mathrm{o}}=c_{\mathrm{o}} N / T$ where $c_{\mathrm{o}}$ is a material constant.

\section{DISCUSSION AND CONCLUSIONS}

The results of the previous section amount to an increase of the relaxation time with particle size. This is indeed observed, for example, in the form of ferromagnetic resonance (FMR) line broadening, although surface contributions have the same effect. ${ }^{9}$ In addition, energy barriers make it difficult to determine $\tau_{\mathrm{o}}$, particularly at low temperatures, where the exponential contribution in Eq. (2) takes over. This affects the determination of both the $N$ and $T$ dependences of $\tau_{\mathrm{o}}$.

The increase of $\tau_{\mathrm{o}}$ with $N$ is limited to nanoparticles, and the formal limit $N=\infty$ has no physical meaning. This is because the exchange in big particles is no longer able to ensure a parallel spin alignment and the straightforward summation leading from Eq. (4) to Eq. (5) can no longer be used. Alternatively, thermal fluctuations give rise to a dynamic spin structure, as shown in Fig. 2. The size $N_{\mathrm{o}}$ of the correlated region is difficult to estimate. Time- and temperaturedependent magnetization measurements (magnetic viscosity and sweep-rate dependence of coercivity) yield thermal activation volumes that contain thousands of atoms. ${ }^{16,19,20}$ From a micromagnetic point of view, the length scale of the magnetization inhomogenities in soft materials (no energy barriers) is of the order of $10 \mathrm{~nm}$, that is, $N_{\mathrm{o}} \sim 10^{4}-10^{5}$. In terms of Sec. II, and taking into account that typical measurements are taken at room temperature, this corresponds to the very rough estimate $c_{\mathrm{o}} \sim 10^{-11} \mathrm{Ks}$.

In conclusion, we have used quasiclassical model calculations and quantum-mechanical arguments to investigate the particle-size dependence of the relaxation-time parameter $\tau_{\mathrm{o}}$. For small particles, the relaxation time is proportional to $N$, but when the particle size becomes comparable to a few nanometers, $\tau_{\mathrm{o}}$ approaches the bulk value. Our calculations elucidate some aspects of magnetic relaxation in nanostructures, but a detailed quantitative description and a comprehensive experimental analysis remain as important challenges to future research.

\section{ACKNOWLEDGMENTS}

This research is supported by AFOSR, DOE, NSF MRSEC, by the Keck foundation, and by CMRA.

${ }^{1}$ G. M. Sandler, H. N. Bertram, T. J. Silva, and T. M. Crawford, J. Appl. Phys. 85, 5080 (1999).

${ }^{2}$ H. Okumura, J. Twisselmann, R. D. McMichael, M. Q. Huang, Y. N. Hsu, D. E. Laughlin, and M. E. McHenry, J. Appl. Phys. 93, 6528 (2003).

${ }^{3}$ H. Mori, Prog. Theor. Phys. 33, 423 (1965).

${ }^{4}$ W. Brenig, Statistical Theory of Heat: Nonequilibrium Phenomena (Springer, Berlin, 1989).

${ }^{5}$ R. Skomski, R. D. Kirby, and D. J. Sellmyer, J. Appl. Phys. 85, 5069 (1999).

${ }^{6}$ R. Skomski, R. D. Kirby, and D. J. Sellmyer, J. Appl. Phys. 93, 6820 (2003).

${ }^{7}$ H. Haken, Synergetics (Springer, Berlin, 1977).

${ }^{8}$ T. Kasuya and R. C. Le Craw, Phys. Rev. Lett. 6, 223 (1961).

${ }^{9}$ A. G. Flores, V. Raposo, J. Iñiguez, L. Torres, and S. B. Oseroff, Phys. Status Solidi A 187, 521 (1987).

${ }^{10}$ J. T. Wang, M. O'Donnell, and H. A. Blackstead, Phys. Rev. B 13, 2044 (1976).

${ }^{11}$ H. Risken, The Fokker-Planck Equation (Springer, Berlin, 1989).

${ }^{12}$ F. Bloch, Phys. Rev. 102, 104 (1956).

${ }^{13}$ W. F. Brown, Phys. Rev. 130, 1677 (1963).

${ }^{14}$ K.-H. Fischer and A. J. Hertz, Spin Glasses (Cambridge University Press, Cambridge, 1991).

${ }^{15}$ H. A. Kramers, Physica (Utrecht) 7, 284 (1940).

${ }^{16}$ R. Skomski, J. Phys.: Condens. Matter 15, R841 (2003).

${ }^{17} \mathrm{~A}$. Aharoni, Introduction to the Theory of Ferromagnetism (Oxford University Press, Oxford, 1996).

${ }^{18}$ A. Würger, J. Phys.: Condens. Matter 10, 10075 (1998).

${ }^{19}$ R. Skomski and J. M. D. Coey, Permanent Magnetism (IOP, Bristol, 1999).

${ }^{20}$ Rare-earth Iron Permanent Magnets, edited by J. M. D. Coey (Oxford University Press, Oxford, 1996). 